\title{
QUEEN'S
UNIVERSITY
BELFAST
}

\section{A randomised controlled feasibility trial of Group Cognitive Behavioural Therapy for people with severe asthma}

Yorke, J., Adair, P., Doyle, A-M., Dubrow-Marshall, L., Fleming, S., Holmes, L., \& Shuldham, C. (2016). A randomised controlled feasibility trial of Group Cognitive Behavioural Therapy for people with severe asthma. Journal of Asthma. https://doi.org/10.1080/02770903.2016.1229335

Published in:

Journal of Asthma

Document Version:

Peer reviewed version

Queen's University Belfast - Research Portal:

Link to publication record in Queen's University Belfast Research Portal

Publisher rights

Copyright 2016 Taylor \& Francis.

This work is made available online in accordance with the publisher's policies. Please refer to any applicable terms of use of the publisher.

\section{General rights}

Copyright for the publications made accessible via the Queen's University Belfast Research Portal is retained by the author(s) and / or other copyright owners and it is a condition of accessing these publications that users recognise and abide by the legal requirements associated with these rights.

Take down policy

The Research Portal is Queen's institutional repository that provides access to Queen's research output. Every effort has been made to ensure that content in the Research Portal does not infringe any person's rights, or applicable UK laws. If you discover content in the Research Portal that you believe breaches copyright or violates any law, please contact openaccess@qub.ac.uk. 
A randomised controlled feasibility trial of Group Cognitive Behavioural Therapy for people with severe asthma

Running title: Feasibility of Group-CBT in severe asthma

Word Count: Abstract $=300 ;$ main paper $=4192$

Key words: psychological, cognitive behavioural therapy, anxiety, depression, quality of life, breathlessness 


\section{Abstract}

Objectives: Evidence for the efficacy of Cognitive Behavioural Therapy (CBT) in asthma is developing but it is not known if this translates to benefits in severe asthma or if a group approach is acceptable to this patient group. This study aimed to assess the feasibility and acceptability of Group-CBT in severe asthma.

Method: This was a two-centre, randomised controlled parallel group feasibility study. Eligible participants (patients with severe asthma and a clinically significant diagnosis of anxiety and/or depression - Hospital Anxiety and Depression Scale (HAD) score greater than 8 for the anxiety or depression sub-scale) received Group-CBT in weekly sessions for eight consecutive weeks and usual care or usual care only. Follow-up was for 16 weeks and end points were: Asthma Quality of Life Questionnaire, Asthma Control Questionnaire, HAD, Dyspnoea-12, EuroQual-5D and EuroQuol-VAS.

Results: 51 patients were randomised: $36 \%$ (51 out of 140) consent rate and 25\% (13/51) attrition at week 16 . Screening logs indicated that study take-up was influenced by patients living long distances from the treatment centre and inability to commit to the weekly demands of the programme. Drop-out was higher in Group-CBT compared due to inability to commit to the weekly programme because of poor health. Participants who contributed to focus group discussions reported that Group-CBT contributed to a better understanding of their illness and related approaches to anxiety management and acceptance of their asthma condition. Although weekly face-to-face sessions were challenging, this was the preferred method of delivery for these participants.

Conclusions: This feasibility study shows that Group-CBT warrants further investigation as a potentially promising treatment option for patients with severe asthma. It has been possible but not easy to recruit and retain the sample. Options for a less demanding intervention schedule, such as less frequent face-to-face visits and the use of webbased interventions, require careful consideration. 


\section{INTRODUCTION}

Severe asthma affects approximately $5-10 \%$ of all patients living with asthma, yet accounts for $50 \%$ of the total costs associated with the condition [1]. Although pathophysiological factors play a role in the experience of severe asthma, it is also influenced by psychological comorbidity; most commonly depression and anxiety (prevalence 22$45 \%$ and $8-26 \%$, respectively) [2-4]. In severe asthma, both depression and anxiety are related to impaired healthrelated quality of life. Depression is specifically related to decreased motivation to self-manage, worse adherence to treatment (estimated non-adherence 30-40\%), and fatal or near fatal asthma attacks, [5,6] perhaps due to disease / severity denial, whilst anxiety is associated with greater healthcare utilization, but fewer life threatening events [7]. Therapies that address the psychological burden of severe disease aimed at enhancing a person's ability to selfmanage, could ameliorate these associated health problems.

Cognitive Behavioural Therapy (CBT) aims to empower people to change previous unhelpful thinking and behaviour patterns that maintain poor self-management approaches [8]. The National Institute for Health and Clinical Excellence (NICE) guidance for the management of anxiety and depression recommends that patients have access to CBT [9] but evidence for its use in cases of asthma is lacking and no studies focus specifically on the pattern of severe asthma $[10,11]$. Studies evaluating the use of CBT in asthma have consisted of individual face-to-face therapy sessions. Possible advantages of group compared to individual face-to-face therapy, may include more time in therapy at less cost, as well as the experience of learning from the struggles and progress of others and the social support offered by group treatment $[12,13]$. Therefore, the aim of this study was to assess the feasibility and acceptability [14] of G-CBT for people with severe asthma, to inform the development of a fully-powered trial.

\section{Objectives:}

1. Assess the feasibility and acceptability of a G-CBT manual for delivery of each session

2. Evaluate recruitment strategies, a screening process for anxiety and depression, and study attrition rates

3. Explore the views of patients regarding G-CBT, trial processes and outcome assessments

4. Assess outcome measures to estimate effect sizes and variability between groups to inform a power calculation for fully powered trial

5. Explore possible hypotheses for a fully powered trial; including effect of G-CBT on anxiety, depression, quality of life and breathlessness.

\section{METHODS}

Appropriate ethical and local governance approvals were obtained (ref: 11/NW/0757). This feasibility study was a randomised, controlled parallel group trial comparing usual care and G-CBT delivered by trained clinical psychology therapists to usual care only.

Patients from two tertiary hospitals in England attending one of a small sub-group of national specialist severe asthma clinics were screened for the following eligibility criteria: adults ( $\geq 18$ years of age) with a confirmed 
diagnosis of severe refractory asthma [15] and receiving standard of care therapy at BTS Steps 4 and 5 level [16]. Patients were routinely screened for the presence of clinically significant anxiety and/or depression using the Hospital Anxiety and Depression scale (HADS) (score $>8$ for anxiety or $>8$ for depression) [17]. Patients with a specific psychiatric condition (e.g. schizophrenia, hypomania) were excluded.

The intervention group received weekly sessions of G-CBT ( 1.5 hours per session) for 8 weeks. The sessions were based on a well-established programme of cognitive-behavioural stress management for severe illness [18,19]. This approach was felt to be most relevant for managing the psychological consequences of severe asthma given its focus on relaxed breathing to combat anxiety related breathlessness. The sessions were guided by a manual to enhance therapist adherence to manual instructions between sites. Instructions for practising relaxation between sessions were given via a compact disc along with a range of relaxation exercises. Personalised goal setting was a fundamental component of the group.

Topics covered included:

1. Relaxed breathing/ Introduction to the CBT model and its application to anxiety and depression in the context of severe asthma

2. Relaxed breathing/Stress and Awareness of asthma exacerbations

3. Relaxed breathing/Linking Thoughts and Emotions

4. Relaxed breathing /Negative thinking and Cognitive Distortions

5. Relaxed breathing/Building resilience/feeling in control/ developing effective coping responses

6. Relaxed breathing/ Developing problem solving skills

7. Relaxed breathing/Effective communication and gaining social support from significant others

8. Relaxed breathing /Coping with setbacks and unscheduled events/Review of the programme

As this was a feasibility study there were no designated primary or secondary outcome measures. Instead, measures were used to determine participant acceptability and variability in order to estimate a sample size for a definitive trial. Participants in both groups were assessed by the research nurse (RN) at baseline, week 8 (completion of intervention), and then at week 16 (end of study).

\section{Outcome assessments}

Asthma Quality of Life Questionnaire (AQLQ): a disease-specific scale composed of 32 questions grouped into 4 domains measuring symptoms, activity limitations, emotional responses, and reactions to environmental stimuli [20]. Scores for each domain, as well as a total composite score, can range from 1 to 7 with higher scores indicating better condition.

Asthma Control Questionnaire (ACQ): requires patients to recall their experiences during the previous week and to respond to the first 6 questions (night-time waking, symptoms on waking, activity limitation, shortness of breath, 
wheeze and rescue short-acting $\beta_{2}$-agonist use) on a 7-point scale [21]. Clinic staff score $\mathrm{FEV}, \%$ predicted preadministration of a bronchodilator on a similar 7-point scale. The ACQ score is the mean of the 7 items: total score between 0 (totally controlled) and 6 (severely uncontrolled).

Dyspnoea-12: quantifies the severity of breathlessness and consists of 12 items each ranging from 0 ('none') to 3 ('severe'). It yields a total score (range 0-36, with higher scores corresponding to greater dyspnoea) and two component scores: physical (includes items 1-7) and affective (includes items 8-12) [22]. The Dyspnoea-12 has demonstrated excellent reliability and validity in asthma [23].

Hospital Anxiety and Depression scale (HADS): a widely used tool for assessing psychological distress (seven items tap anxiety with a score range of $0-21$, and seven items tap depression with a score range of $0-21$; higher scores indicate greater emotional distress) [17]. HADS anxiety score $>8$ represents symptoms of anxiety and a HAD depression score $>8$ represents symptoms of depression.

The EuroQual-5 Dimension (EQ-5D-5L): is a standardised instrument for use as a measure of health outcome including mobility, self-care, usual activities, pain/discomfort and anxiety/depression [24]. EQ-5D scores range between -0.594 and 1 (full health). In addition, there is a visual analogue scale (VAS) to indicate the general health status with 100 indicating the best health status.

Exacerbation frequency and health care utilization using asthma diary: participants were provided with a specifically developed asthma diary for this study. Participants were requested to record each episode for exacerbations and access to health care practitioner (including accident and emergency visits, hospital admissions and consults with nurses, doctors, and other health care workers).

Focus groups and one-to-one interviews were conducted with participants (both intervention and control group) to elicit views regarding intervention acceptability, use of outcome measures, and trial conduct. A semi-structured format was used to ensure key themes were covered [25] and all were digitally recorded and transcribed verbatim.

As this was a feasibility study there was no formal sample size calculation, although the usual convention for feasibility studies [26] is for approximately 30/arm. Recent studies suggest smaller samples sizes of 15/arm (after attrition, for medium effect sizes with $90 \%$ power) can be justified [26]. Therefore it was intended to randomise approximately $30 /$ arm to allow for an unknown attrition rate in this population.

Prior to consenting, patients were requested to complete the HADS to determine the presence of anxiety and/or depression. Following consent, participants were requested to return to the hospital for a study visit to complete baseline assessments and randomisation (1:1 ratio). Randomisation was carried out independently by the Clinical Trials and Evaluation Unit (CTEU), Royal Brompton and Harefield National Health Service Foundation Trust 
(RBHT). Data collection-design facilitated RN-blinding to group allocation however, this was difficult to maintain as patients often discussed their treatment with the $\mathrm{RN}$ at subsequent study follow-ups.

\section{Data analysis}

The SPSS (version 22.0) statistical computer package was used for analysis [27]. The AQLQ sub-scales and total scores, ACQ, HAD anxiety and depression scores, Dyspnoea-12 Physical, Affective and Total, and EQ-5D scores were analysed by calculating a paired difference (eight weeks-baseline and 16 weeks-baseline) and the paired differences were compared between the two randomised groups using an independent t-test. Seven subjects ( $3 \mathrm{G}$ CBT and 4 control) who undertook the study were later found not to meet the inclusion criteria, and were removed from all analyses, leaving 44 (from 51 randomised). Participants allocated to receive G-CBT but withdrew prior to starting treatment $(n=3)$ or provided baseline data only ( $n=2$; attended 2 or less sessions) were removed from further analyses. As this was a feasibility study, the statistical differences between the groups were of much less importance than determining variability for a planned larger study [28]. Effect size was calculated as the ratio between the estimated mean group difference $\left(\bar{\Delta}_{\text {wk16-wk0 }} \mathrm{CBT}-\bar{\Delta}_{\text {wk16-wk0 }}\right.$ Control $)$ and the pooled standard deviation of differences (SD) (also known as the standardised difference) [28]. No adjustment for multiple comparisons was made.

The distribution of change scores between the Group-CBT and control groups was examined for each outcome to identify a potential end-point for a future trial. The minimal clinical important difference (MCID) was then determined and used to calculate a power calculation and sample size for a future trial. The minimally clinically important difference (MCID) is commonly reported as the change in scores on the measurement scale in question that relates to the smallest change in symptom intensity subjectively recognized by the patient. The MCID for any particular patient reported outcome measure can be determined using distribution-based calculation (i.e. effect size = mean/sd) and anchor-based methods (i.e. correlation, linear regression, ROC analysis). ROC analysis considers how accurately two populations can be discriminated from each other, by creating a single value (area under the curve) based on every possible cut-point between the two groups. High values of the AUC (near the maximum of 1 ) represent good discrimination, whilst low values (near the minimum of 0.5 ) are poor [29-31].

Qualitative data were anonymised and analysed thematically [25] Codes were placed under the relevant themes, for example: 'views', 'preferences', and 'expectations', with additional themes constructed as necessary. Codes and themes were extracted independently by two researchers (JY and SF) and compared for consistency.

\section{RESULTS}

The aims of this study were to assess feasibility of the intervention and trial processes, and to look for variability in the outcome measures to power a larger RCT. As such, summary statistics of the data are presented and not estimates of significance. 


\section{Recruitment}

140 patients were deemed to have met the inclusion criteria and were approached by the clinician and/or research nurse to take part, and 51 (36\%) consented and were randomised between March 2012 and November 2012 to one of the two study arms (Figure 1). The main reasons for not consenting to participate was due to travelling concerns including living too far from the treatment site or not having access to transportation $(35 \%$ of those approached, $\mathrm{n}=$ 32; 30 from central London site), family commitments $(n=24,27 \%)$ and work commitments $(n=19,21 \%)$. Figure 1 illustrates the reasons for patients declining to take part.

\section{Data completeness}

The CONSORT flow diagram (Figure 1) shows the flow of participants through the trial. There were seven protocol deviations due to error - seven patients were entered into the trial who had a HAD score eight or less for anxiety or depression (three were randomised to G-CBT of which two dropped out pre-treatment and four in the control group); all were removed from further analyses. For each variable there was less than $10 \%$ missing data. The only exception was asthma diaries (discussed below). This leaves 44 in the analysis dataset. Attrition at week 16 was 12 (intervention group $n=10$ and control group $n=2$ ).

\section{Demographics and baseline characteristics}

The average age of the patients was 46.7 years (range 20-70); 25 (57\%) were women. Baseline characteristics for the entire sample are shown in Table 1. The baseline characteristics were compared between those who reached week16, and those who did not to assess if there had been any potential bias due to drop-outs in each treatment group. There did not seem to be any differential attrition associated with any of the baseline characteristics, except perhaps for previous therapy - although there was too much missing data to draw clear conclusions. All potential participants were screened for psychological co-morbidity with the HADS. Fifteen potential participants reported a low HADS score and were excluded from participation. The mean anxiety and depression scores for all eligible consented participants ( $n=44$ ) was 12.8 (SD 3.5; range 7 to 20) and 10.7 (SD 3.8; range 3 to 17), respectively.

Insert Table 1

\section{G-CBT attendance}

Of the 23 eligible participants who commenced G-CBT, four attended each of the eight sessions and a further eight attended between five and seven sessions. The main reason for missing sessions was being too unwell with their asthma to travel or actively participate in the session. One participant was receiving intravenous asthma therapy as an in-patient during the first three sessions but still attended each of the G-CBT sessions at the time.

\section{Asthma diaries}

The diaries were completed by 14 participants (G-CBT 7 and control 7), all of whom completed week 16 follow-up. Two G-CBT and one control participant reported an accident and emergency asthma-related visit and all resulted in 
a hospital admission. General Practitioner visits were recorded four times for G-CBT participants and eight for the control group. Contact with asthma specialists (nurse or doctor either by phone or in a face-to-face consultation) were recorded four times for G-CBT and nine times for the control group. Diary completion in each group was insufficient to conduct meaningful comparisons.

\section{Outcomes assessments}

We conducted data analysis for outcomes at week 16 assessment for trial completers (G-CBT $n=17$ and control $n$ $=20$ ). As a general pattern, effect sizes were small and mostly favoured the treatment group (table 2 and figure 2). The G-CBT group appeared to do better with regard to AQLQ subscales for symptoms and emotions, asthma control, breathlessness severity (particularly the D-12 affective sub-scale), and anxiety; but not for AQLQ Total and its subscales activity and environment. Figure 2 demonstrates variability of the scores for each outcome measure at baseline, week 8 and week 16, with a trend in scores to show more improvement in the G-CBT arm at week 16. The most meaningful assessment point was observed at week 16 (figure 2) and was used as the endpoint for data analysis. Dyspnoea-12 was chosen as the primary outcome to base the sample size calculation for a follow-on trial because it was significant for the G-CBT group at week 16 had a large effect size of 0.62 .

Insert table 2 and Figure 2

\section{MCID and sample size calculations}

Using distribution-based analyses using effect sizes [29] both D12 and HADS were identified as potential endpoints for a future trial, but only D12 showed a large improvement in the treatment group (Table 2). Using anchor-based methods, we examined the correlation between change in AQLQ and D12 [29-31] and showed that with a Pearson's correlation of -0.45 that D12 was suitable as an endpoint (i.e. $>0.3$ ). We considered the regression between these variables [29] which suggested an MCID for D12 of at least 2.0. Using receiver operating characteristics (ROC) methods [29], a binary status was created using from $\triangle A Q L Q$, where those with for $\triangle A Q L Q<-$ 0.208 are "worsening" and those with $\triangle A Q L Q>0.208$ are "improving (standard error of the mean of $\triangle A Q L Q$ was 0.208 ). Although this left only $n=22$ observations (the rest were "stable") this analysis suggested the MCID for D12 should be $\geq 2.5$ (area=0.74). Synthesing the distribution-based \& anchor-based evidence, it was decided that an MCID for D12 of 3.5 was justified.

Table 2 shows the standardised difference (effect size) for each of the outcome measures. The difference in change scores in Dyspnoea-12 between the G-CBT and control groups was observed to be large (G-CBT: -5.5 , Control: -0.1) and consistent between the groups demonstrating that the future effect will be in only direction and the use of 1 -sided t-test for powering a full trial was justified. Dyspnoea-12 was used to determine sample size for a follow-on trial, and a sample size of 74 per arm has $80 \%$ power at week 16 to detect a minimal clinically important 
difference of 3.5 units using a 5\% significance level (assuming a CBT SD of 6.6 and Control SD of 10.0). There were $73 \%$ (32/44) retained at week 16 giving an attrition rate of $27 \%$, but a change to decrease the number of intervention face-to-face sessions should reduce this by at least $1 / 3$ to $18 \%$. Therefore $91 /$ arm (182 in total) should be recruited.

\section{Participant views}

We conducted four focus groups with 10 participants and two one-to-one interviews with participants who received G-CBT. See table 3 for pertinent quotes. All participants who took part in the focus groups and received the intervention reported that they liked being part of a group and for some hearing others speak of similar problems to their own helped to normalise their own experiences. Some participants suggested that they would not disclose certain issues if therapy was one-to-one as sharing experiences with other people with severe asthma provided an environment where things could be discussed openly. Participants stated that they would rather focus on asthma issues than more generic stress management techniques as that is what they found most useful. Most participants found the relaxation and the learning of different ways to "switch off" to be the most beneficial. All stated that the 8 sessions were needed; some wanted more sessions or at least some sort of follow-up group. The timing of the group sessions was difficult for some, and travelling a long distance on a weekly basis was extremely challenging. Focus group participants agreed that face-to-face sessions in a group format were preferred to one-to-one sessions or sessions in any format, using telephones and web-based sources including Skype.

\section{DISCUSSION}

This study demonstrates the importance of a feasibility stage in trials evaluating complex interventions - especially in patients with a severe and life threatening condition. We found that recruitment is challenging but possible from this patient group. To the authors' knowledge, this is the first randomised controlled trial of CBT to focus on people with severe asthma, and the first to randomised trial of a group-based psychological intervention in any asthma population. We can conclude that group therapy is acceptable to some patients with severe asthma - those who completed this study and participated in the follow-up focus groups; but the intervention in its current form has too high an attrition rate to be beneficial in wider clinical practise. In support of previous research, many participants found the relaxation techniques as being particularly helpful in preventing panic-related episodes and breathlessness easing [32]. Important strengths and limitations have been identified and require careful consideration in protocol development for the follow-on trial.

Recruitment was less than expected. Patients declined to take part due to travelling requirements and commitments relating to family and work. Of note, 30/32 patients who declined to participate due to travelling were located at the London site where transport to the hospital is largely dependent on the London underground tube, in comparison to car or bus travel for the other site. This may have influenced people's willingness to make the weekly journey for the intervention. This issue is important as regional centres of excellence were used, who have patient populations with wide catchment geography. The distance required to travel for some participants was approximately two hours each 
way - the stress of travelling each week may have been perceived as too burdensome and time consuming. Participants completing the course who were also required to travelling distances were more likely to commute by private car. Attrition was higher in G-CBT compared to the control group with three participants withdrawing prior to the commencement of G-CBT and a further two during the active treatment period. The reasons for withdrawal varied with being 'too ill' the main one cited. Similar recruitment and retention difficulties have been previously reported in trials of one-to-one CBT in asthma [10,11]. Attendance at group sessions was also variable. This raises the question about commitment to non-pharmacological studies by some patients. Low attendance may also represent avoidance coping styles whereby some people may not feel that they would benefit from such therapies [11], despite HAD scores that are indicative of clinically relevant anxiety and/or depression. There were no significant differences in clinical variables or study outcome assessments in G-CBT completers and noncompleters, suggesting that factors other than clinical variables influence study attrition.

Weekly sessions may have contributed to the high drop-out rate in the intervention group compared to the control group although an active control group also attending weekly study visits would be required to test this hypothesis. This was a feasibility study and was not designed to test the effectiveness of the intervention, therefore we did not control for attention but a future trial should consider this important issue. Focus group participants suggested that weekly visits were important for continuity however we do not have the views and expectations of participants who dropped out or did not consent to take part. In general, patients were committed to accessing psychological support but the burden of weekly attendance was a deterrent for some. This factor needs to be considered in the development of a future trial offering a range of options to patients perhaps including the use of web-based technology to lessen burden. This would require further patient involvement in the delivery of such interventions and careful screening for suitable participants; given that those patients who completed the face-to-face sessions valued the interaction with other people with severe asthma and stated that this was the preferred mode of intervention delivery compared to web-based therapy.

Online CBT is a proven viable and effective alternative to face to face treatment with online treatment adherence reported at $82 \%$ and results in reductions in psychopathology in a variety of mental health disorders ${ }^{\text {ref }}$ However, online CBT is yet to be tested in severe asthma. Online delivery may be suited to patients who may be restricted by travel concerns although the feasibility and acceptability of this mode of treatment delivery would require further assessment. The focus group participants in our study consistently advised that online delivery would not be a preferred option for them - favouring a face-to-face group format. This is evidence by the quotes found in Box 1; although only patients who signed-up for group face-to-face therapy were interviewed.

This study aimed to determine the effect size of the assessments used, allowing calculation of the numbers needed for an appropriately powered study. However, caution is required when interpreting the statistical results due to the small sample size. Using the standardised difference approach breathlessness severity, measured using the 
Dyspnoea-12 questionnaire, demonstrated the largest effect size with an achievable sample size for a future study. Other outcome measures including AQLQ, ACQ and HAD, did not appear to be as discriminatory. This study was designed to assess feasibility of Group-CBT and not to test its effectiveness - the study was underpowered. This may account for the small effect sizes found in study outcomes. Lack of control for attention meant that the intervention group received significantly more attention so the small effect sizes for anxiety and depression are not clear. Although the reasons for this are not entirely clear it may represent the importance of dyspnoea in the severe asthma population.

The Dyspnoea-12 uses descriptor items, known to be relevant to patients with asthma, to quantify breathlessness severity. Breathlessness is an important outcome in asthma since it provides the patient with an alarm signal, making a vital component of self-monitoring $[1,23]$. Breathlessness is multidimensional composed of at least a physical sensation and emotional reaction [22]. The affective component of breathlessness evokes distress and motivates health behaviours, making it a key outcome of symptom targeted therapies. The Dyspnoea-12 measures both physical and affective components of breathlessness. Post hoc analyses found that Dyspnoea-12 affective component scores improved significantly at week 16 compared to the control group. In the Dyspnoea-12 asthma validation study, Yorke et al. reported that the affective component had stronger correlations with the HAD questionnaire than the physical component [23]. Dyspnoea-12 scores reported in the current study of patients with severe asthma are notably higher than those reported in the Dyspnoea-12 validation study conducted in people with mild-moderate asthma. This suggests that breathlessness plays an important role in severe asthma, and possibly more so than people with mild-moderate asthma. In addition, our qualitative work indicated that the relaxation and breathing components of the CBT programme were highly valued by participants. Some G-CBT participants stated that these techniques helped them to gain better control of their anxiety and breathing, suggesting the Dysnoea-12 captures this in a way that is not provided for with the other outcome measures used.

Previous studies evaluating the effectiveness of individual CBT in the general asthma population have found the AQLQ $[10,11,33,34]$ to be sensitive to change. Although this study was not powered to detect significant differences between G-CBT and control groups, the negative results for these outcomes is somewhat surprising. It is, however, important to note that no previous CBT study has targeted severe asthma patients or evaluated groupbased therapy [10]. It may be that in severe disease the experience of asthma patients and their expectations of improvement from non-pharmacological interventions differ from the general asthma population. The side-effects of steroids and intensive treatment regimen were perceived by our study patients as being significant - yet some reported a more positive outlook in regards to treatment following the G-CBT. Concerns about asthma medicines and side-effects are known to add to the burden of living with asthma and lower levels of medication adherence [35]. The outcome measures used in the current study do not tap these important domains of living with severe asthma. The AQLQ and the ACQ provide important information about the experienced symptoms, activity limitations, emotional functioning, and impact of environmental stimuli on these patients. However, health status in severe asthma most certainly comprises of more sub-domains than the four subscales covered by the AQLQ and 
the one subscale of the ACQ. An outcome measure that provides a more detailed and comprehensive picture of the impact of asthma in severe disease may be more useful in trials evaluating psychological oriented interventions.

This study has limitations. Access to the trial was available to patients receiving treatment for severe asthma at only two specialist clinics in the United Kingdom and there for generalizability of the results is limited. However, it should be noted that one clinic was situated in London and the other in the North West where travel by patients to reach the each centre is different; this provided useful feasibility data were provided. We conducted focus groups at trial completion to ascertain participants' views in relation to study processes and aspects of the intervention. Due to ethical restrictions, participants who opted not to take part or dropped out of the study were not interviewed. Therefore only the views and opinions of active participants were explored. This is likely to account for the limited support offered during focus groups for alternate intervention delivery modes such as online. This study did not control for attention - the intervention group received significantly more attention compared to the control group. It is known that participating in a group setting and interactions with research staff can improve study outcomes. Whilst this is an important consideration the current study was a feasibility study and not designed to determine the effectiveness of the intervention.

\section{Conclusion}

This feasibility study shows that G-CBT warrants further investigation as a potentially promising treatment option for patients with severe asthma; although careful assessment and consideration of participant commitment would need to be included. It has been possible but not easy to recruit and retain the sample. Careful thought would need to be given to issues of commitment and timing, and model of treatment delivery for participation in the follow-on study. The results indicate that a comprehensive assessment of severe-asthma related quality life is warranted and that the Dyspnoea-12 would be an appropriate endpoint, with a manageable sample size estimated. However, this would require multi-site collaboration to achieve the numbers needed. The follow-on study should include health economic evaluation and continued qualitative exploration of the experience of study participants receiving the intervention. References

1. O'Neill S, Sweeney J, Patterson CC, Menzies-Gow A, Niven R, Mansur AH, Bucknall C, Chaudhuri R, Thomson NC, Brightling CE, O'Neill C, Heaney LG; on behalf of the British Thoracic Society Difficult Asthma Network. The cost of treating severe refractory asthma in the UK: an economic analysis from the British Thoracic Society Difficult Asthma Registry. Thorax 2014 Jun 10. pii: thoraxjnl-2013-204114. doi: 10.1136/thoraxjnl-2013-204114. [Epub ahead of print].

2. Heaney LG, Robinson DS. Severe asthma treatment: need for characterising patients. Lancet. 2005;365(9463): 974-976.

3. Mancuso CA, Peterson MG, Charlson ME. Effects of depressive symptoms on health related quality of life in asthma patients. J Gen Intern Med. 2000; 15:301-310. 
4. Lavoie KL, Bouthillier D, Bacon SL. Psychologic distress and maladaptive coping styles in patients with severe versus moderate asthma. Chest 2010;137(6): 1324-1331.

5. Gamble J, Stevenson M, McClean E, Heaney LG. The prevalence of non-adherence in difficult asthma. Am J Respir Crit Care Med. 2009;180: 817-822.

6. Schneider A, Lowe B, Meyer FJ, Biessecker K, Joos S, Szecsenyi J. Depression and panic disorder as predictors of health outcomes for patients with asthma in primary care. Respir Med. 2008;102(2): 566-574.

7. Kullowatz A, Kanniess F, Dahme B, Magnussen H, Ritz T. Association of depression and anxiety with health care use and quality of life in asthma patients. Respir Med. 2001;101: 638-644.

8. British Association for Behavioural and Cognitive Psychotherapies. What are Cognitive and/or Behavioural Psychotherapies: Paper prepared for a UKCP/BACP mapping psychotherapy exercise by Katy Grazebrook, Anne Garland and the Board of BABCP. 2005; Retrieved on 21-01-2011.

9. National Institute for Clinical Excellence. CG22 Anxiety: management of anxiety (panic disorder, with or without agoraphobia, and generalised anxiety disorder) in adults in primary, secondary and community care. National Institute for Clinical Excellence Guidelines. 2004.

10. Yorke J, Fleming SL, Shuldham C. Psychological interventions for adults with asthma. Cochrane Database of Systematic Reviews. 2006; 1: CD002982. DOI: 10.1002/14651858.CD002982.pub3.

11. Parry GD, Cooper CL, Moore JM, Yadegarfar G, Campbell MJ, Esmonde L. Cognitive behavioural intervention for adults with anxiety complications of asthma: prospective randomised trial. Respir Med. 2012;106[6]: 802-10.

12. Tucker M, Oei TPS. Is Group More Cost Effective than Individual Cognitive Behaviour Therapy? The Evidence is not Solid Yet. Behavioural and Cognitive Psychotherapy. 2007;35: 77-91.

13. O'Dowd H, Gladwell P, Rogers CA, Hollinghurst S, Gregory A. Cognitive behavioural therapy in chronic fatigue syndrome: a randomised controlled trial of an outpatient group programme. Health Technology Assessment 2006;10:3.

14. Arain M, Campbell MJ, Cooper CL, Lancaster GA. What is a pilot or feasibility study? A review of current practice and editorial policy. BMC Medical Research Methodology 2010, 10:67.

15. American Thoracic Society. Proceedings of the ATS Workshop on Refractory Asthma Current Understanding, Recommendations, and Unanswered Questions. Am J Respir Acute Crit Care Med. 2000;162(6): 2341-51.

16. British Thoracic Society Scottish Intercollegiate Guidelines Network. British guideline on the management of asthma. Thorax 2008;63(Suppl. 4):1e121.

17. Zigmond AS, Snaith RP. The hospital anxiety and depression scale. Acta Psychiatr Scand. 1983;67(6): 36170.

18. Antoni, M.H. Stress Management Intervention for Women with Breast Cancer. 2003. Washington D.C.: American Psychological Association Press.

19. Antoni MH, Ironson G, Schneiderman N. Stress Management for Persons with HIV Infection. 2007, New 
York : Oxford University Press.

20. Juniper EF, Guyatt GH, Ferrie PJ, Epstein RSEvaluation of impairment of health related quality of life in asthma: development of a questionnaire for use in clinical trials. Thorax. 1992;47: 76-83.

21. Juniper EF, O'Byrne PM, Guyatt GH, Ferrie PJ, King DR. Development and validation of a questionnaire to measure asthma control. Eur Respir J. 1999;14: 902-907.

22. Yorke J, Jones PW, Swigris J. Development and validity testing of an IPF-specific version of the SGRQ (the SGRQ-I). Thorax. 2010; 65:921-926.

23. Yorke J, Russell A, Swigris J, Shuldham C, Haigh C, Jones, PW. Assessment of dyspnoea in asthma: validation of the Dyspnoea-12. J Asthma. 2011;48(6): 602-08.

24. Rabin R, de Charro F. EQ-5D: a measure of health status from the EuroQol Group. Ann Med 2001;33(5):337-43.

25. Green J \& Thorogood N. Qualitative Methods for Research. Sage, 2004, London.

26. Whitehead AL, Julious SA, Cooper CL, Campbell MJ. Estimating the sample size for a pilot randomised trial to minimise the overall trial sample size for the external pilot and main trial for a continuous outcome variable. Stat Methods Med Res. 2015.

27. IBM Corp. Released 2013. IBM SPSS Statistics for Windows, Version 22.0. Armonk, NY: IBM Corp.

28. Browne R.H. (1995) On the use of a pilot sample for sample size determination. Statistics in Medicine 14 , 1933- 1940

29. Oxberry SG, Bland MJ, Clark AL, Cleland JGF, Johnson M. Minimally clinically important difference in chronic breathlessness : Every little helps. Ame Heart J 2012;164(2):229-35.

30. de Kleijn WP, De Vries J, Wijnen PA, Drent M. Minimal (clinically) important differences for the Fatigue Assessment Scale in sarcoidosis. Respir Med 2011;105:1388-1395.

31. Johnson MJ, Bland JM, Oxberry SG, Abernethy AP, Currow DC. Clinically important differences in the intensity of chronic refractory breathlessness. J Pain Symptom Manage 2013;46(6):957-63.

32. Holloway EA, West RJ. Integrated breathing and relaxation training (the Papworth method) for adults with asthma in primary care: a randomised controlled trial. Thorax 2007;0:1-5.

33. Grover N, D'Souza, G., Thennarasu, K., Kumaraiah, V. Randomised controlled study of CBT in bronchial asthma. Lung India. 2007;24:45-50.

34. Ross CJ, Davis TM, MacDonald GF. Cognitive-behavioral treatment combined with asthma education for adults with asthma and coexisting panic disorder. Clin Nurs Res. 2005; 14:131-57.

35. Cochrane GM, Horne R, Chanez P. Compliance in asthma. Respir Med. 1999;93: 763-769. 
Table 1: Baseline data: demographics and clinical characteristics

\begin{tabular}{|c|c|c|c|c|}
\hline & $\begin{array}{c}\text { G-CBT (all } \\
\text { participants) } \\
n=23\end{array}$ & $\begin{array}{c}\text { Control (all } \\
\text { participants) } \\
n=21\end{array}$ & $\begin{array}{c}\text { Drop-out at } \\
\text { week16 } \\
\mathrm{n}=12\end{array}$ & $\begin{array}{c}\text { Completer to } \\
\text { week16 } \\
n=32\end{array}$ \\
\hline $\begin{array}{l}\text { Age: mean (SD, } \\
\text { range) }\end{array}$ & $48.6(11.1,22-63)$ & $45.0(13.7,20-70)$ & $46.4(11.2,22-63)$ & $47.0(13.0,20-70)$ \\
\hline Gender $\mathrm{n}(\%$ female) & $15(65)$ & $10(48)$ & $8(67)$ & $17(53)$ \\
\hline $\begin{array}{l}\text { Ethnicity } \mathrm{n}(\%) \\
\text { White British } \\
\text { White other } \\
\text { Asian }\end{array}$ & $\begin{array}{l}21(91) \\
2(9) \\
0(0)\end{array}$ & $\begin{array}{c}19(91) \\
1(5) \\
1(5)\end{array}$ & $\begin{array}{c}11(92) \\
1(8) \\
0(0)\end{array}$ & $\begin{array}{c}29(91) \\
2(6) \\
1(3)\end{array}$ \\
\hline $\begin{array}{l}\text { Previous counselling / } \\
\text { talking therapy }(\% \\
\begin{array}{l}\text { yes) } \\
\end{array}\end{array}$ & $\begin{array}{c}5(50) \\
13\end{array}$ & $\begin{array}{c}4(50) \\
13\end{array}$ & $\begin{array}{c}1(20) \\
7\end{array}$ & $\begin{array}{c}8(62) \\
19\end{array}$ \\
\hline $\begin{array}{l}\text { HAD Anxiety: } \\
\text { mean(SD) }\end{array}$ & $12.1(3.8)$ & $13.6(3.0)$ & $13.0(3.5)$ & $12.8(3.5)$ \\
\hline $\begin{array}{l}\text { HAD Depression: } \\
\text { mean (SD) }\end{array}$ & $10.0(4.0)$ & $11.5(3.4)$ & $10.3(4.1)$ & $10.9(3.7)$ \\
\hline ACQ: mean (SD) & $3.3(1.0)$ & $3.6(1.0)$ & $3.4(1.2)$ & $3.5(1.0)$ \\
\hline \multicolumn{5}{|l|}{ AQLQ: mean (SD) } \\
\hline Total & $3.5(1.1)$ & $3.1(1.2)$ & $3.3(1.4)$ & $3.3(1.1)$ \\
\hline Symptoms & $3.3(1.2)$ & $2.8(1.3)$ & $3.3(1.5)$ & $3.0(1.0)$ \\
\hline Activity & $3.6(1.1)$ & $3.3(1.2)$ & $3.4(1.4)$ & $3.5(1.1)$ \\
\hline Emotions & $3.2(1.2)$ & $2.8(1.3)$ & $3.0(1.1)$ & $3.0(1.3)$ \\
\hline Environment & $4.0(1.7)$ & $3.8(1.6)$ & $3.6(1.7)$ & $4.0(1.6)$ \\
\hline $\begin{array}{l}\text { D-12: mean (SD) } \\
\text { Total }\end{array}$ & $24.1(8.6)$ & $23.5(9.2)$ & $23.8(8.7)$ & $23.8(9.0)$ \\
\hline Euor-Qual Index & $0.49(0.26)$ & $0.45(0.32)$ & $0.44(0.28)$ & $0.48(0.29)$ \\
\hline Euro-Qual VAS & $47.2(16.4)$ & $50.0(22.2)$ & $46.7(16.3)$ & $49.2(20.4)$ \\
\hline $\mathrm{FEV}_{1} \%$ predicted & $61.1(35.1)$ & $56.8(30.4)$ & $63.5(21.9)$ & $58.3(34.2)$ \\
\hline
\end{tabular}

G-CBT: group cognitive behavioural therapy; SD: standard deviation; BTS: British Thoracic Society; AQLQ: Asthma Quality of Life Questionnaire; ACQ: Asthma Control Questionnaire; D-12: Dyspnoea-12; HAD: hospital anxiety and depression scale; EQ-5D: EuroQuol Five Dimensions; VAS: visual analogue scale. 
Table 2: Analysis of outcome measures:

baseline to week 16

\begin{tabular}{|c|c|c|c|c|}
\hline \multirow[t]{2}{*}{ Outcome measure } & \multicolumn{4}{|c|}{ Mean change (Week16-baseline) } \\
\hline & $\begin{array}{l}\text { G-CBT } \\
\text { mean (SD) } \\
\mathrm{n}=13\end{array}$ & $\begin{array}{l}\text { Control } \\
\text { mean (SD) } \\
\mathrm{n}=18-19\end{array}$ & $\begin{array}{l}\text { Effect } \\
\text { size }\end{array}$ & $\begin{array}{l}\text { t-test } \\
p \text {-value }\end{array}$ \\
\hline AQLQ & & & & \\
\hline Total & $-0.2(1.5)$ & $0.2(0.9)$ & 0.34 & 0.357 \\
\hline Symptoms & $-0.1(1.5)$ & $0.2(1.0)$ & 0.23 & 0.531 \\
\hline Activity & $-0.2(1.4)$ & $0.2(1.0)$ & 0.33 & 0.371 \\
\hline Emotions & $-0.1(1.8)$ & $0.2(1.6)$ & 0.20 & 0.591 \\
\hline Environment & $-0.6(1.8)$ & $0.5(1.3)$ & 0.72 & 0.056 \\
\hline$A C Q$ & $-0.2(1.0)$ & $0.2(1.1)$ & 0.33 & 0.367 \\
\hline D-12 Total & $-5.5(6.6)$ & $-0.1(10.0)$ & 0.62 & 0.093 \\
\hline D-12 Physical* & $-3.0(4.1)$ & $0.0(4.9)$ & 0.66 & 0.045 \\
\hline D-12 Emotional & $-2.5(3.6)$ & $0.1(5.9)$ & 0.51 & 0.166 \\
\hline HAD Anxiety & $-2.0(3.2)$ & $-2.6(4.7)$ & 0.14 & 0.702 \\
\hline HAD Depression * & $-2.3(3.0)$ & $-1.7(4.1)$ & 0.16 & 0.343 \\
\hline EQ-5D Index** & $0.04(0.07)$ & $0.02(0.30)$ & 0.14 & 0.821 \\
\hline EQ-5D VAS & $5.6(24.1)$ & $-5.4(21.4)$ & 0.49 & 0.185 \\
\hline
\end{tabular}

*Mann-Whitney U test, due to non Normaility

${ }^{\star *}$ Equal variances not assumed

AQLQ: High score=Good. Therefore wk16-wk0 $>0$ is improvement.

ACQ: High score=Bad. Therefore wk $16-w k 0<0$ is improvement 
D12: High score=Bad. Therefore wk16-wk0<0 is improvement

HADS: High score $=$ Bad. Therefore $w k 16-w k 0<0$ is improvement

EQ5D: High score=Good. Therefore $w k 16-w k 0>0$ is improvement. 


\section{Being part of a group:}

Yes, because I think within the group we've called on resources, and all of us in some way, well we've helped each other, but we've all got the common denominator and, you know, you could talk openly about it and that was good, you know, I felt it did me good. (Female, site 2)

It was nice to hear other people because you feel like, it's not all just happening to you and the symptoms that you do suffer...and ideas of how to cope and deal with things as well. I've learned a lot from that, it was quite good. (Female, site 2)

You've had the opportunity to ask other people questions, whereas, if I had done it on my own, I would still be more anxious. (Female,site1)

Talking to people and listening to other people and then, obviously seen people that's in a worse place, I mean there's people...and you just think, pull yourself together sort of thing. Yes, I've got an illness, but you've got to learn to deal with things a bit better and try not to let things get on top of you the same. (Female, site 1)

Yes, because you're all comparing aren't you, and all listening to each other's. Like I say, if I had just come in and it had just been one to one, I wouldn't have opened up like I have done, I don't think. (Female site 1)

\section{Asthma specific issues:}

One of the things was about the treatment that, because you do get a bit worried about the medication side of it...sometimes the treatment is quite aggressive really and quite strong stuff and it was very reassuring to meet other people that do the same. (Female, site 2)

If somebody brought up a problem and said, "I'm really struggling with this and it affects my asthma", then they [therapist] would focus on that and help you to deal with strategies to cope with specific problems with your asthma, and I felt that the asthma became more central and it was more tailored to peoples actual issues rather than just generic, you know, kind of stress. (Female, site 2)

Well, the breathing, you know, control the breathing a bit more...think about your breathing, which I've tried to do more (Female, site 1)

\section{Learning coping strategies:}

There was relaxation; there was talk about the way you think about a situation instead of just being rather buried by it. (Female, site 2 )

And the goals, giving goals, things to aim at...terribly useful (Female, site 2)

I liked the goal setting, trying not to set your goals too high, do too much. (Male, site 1)

\section{Logistics:}

The journey for me really, just a long way to come. Especially when, you know, like today I'm here and then I've got to come back again on Monday to see [the doctor] (Female, site 1)

"Just to do a couple of hours per week, you know, I felt I wanted to get out of the house, get something, where I can tlk to people" (Site 1, F)

"Like I say, it got me out, it's the only day, would want it 7 days really" site $1, F$ )

"Yes, like me, I'm getting, I have a shave and things to come and me appearance is getting a little better...my misuses said "is he going out again?"...can see me getting out, l'm going for help". (Site 1, M).

Regarding having a break midway: “...just getting into it, you know, then a break, no”. (site $1 \mathrm{~F}$ ).

\section{Different options for treatment delivery}

(Intervention delivery over the phone) I don't like that, I don't like speaking on the phone anyway. (Female, site 1)

I feel daft talking to a screen like that, no, I don't do Skype anyway, but no, I mean, if you were to ring me on the phone and ask me how I

were, I would be all right, because I know you, but if it was somebody different, I probably wouldn't be the same. (Female, site 1)

I mean, personally like you say, I prefer a group, where you, you know, you come back and you see the people that you've been with all through the CBT and that would be better really. (Female, site 1)

\section{Maintenance therapy:}

Perhaps two to three weeks, a month later, have us all because we, we'd just got into it really (Female, site 2)

Perhaps once a month [maintenance sessions], yes, like where we had the follow-ups [study appointments] for the questionnaires and the lung tests, perhaps, you know, just a meeting then to see how everybody is getting on (Male, site 1)

Need a refresher course (Female, site 1). 

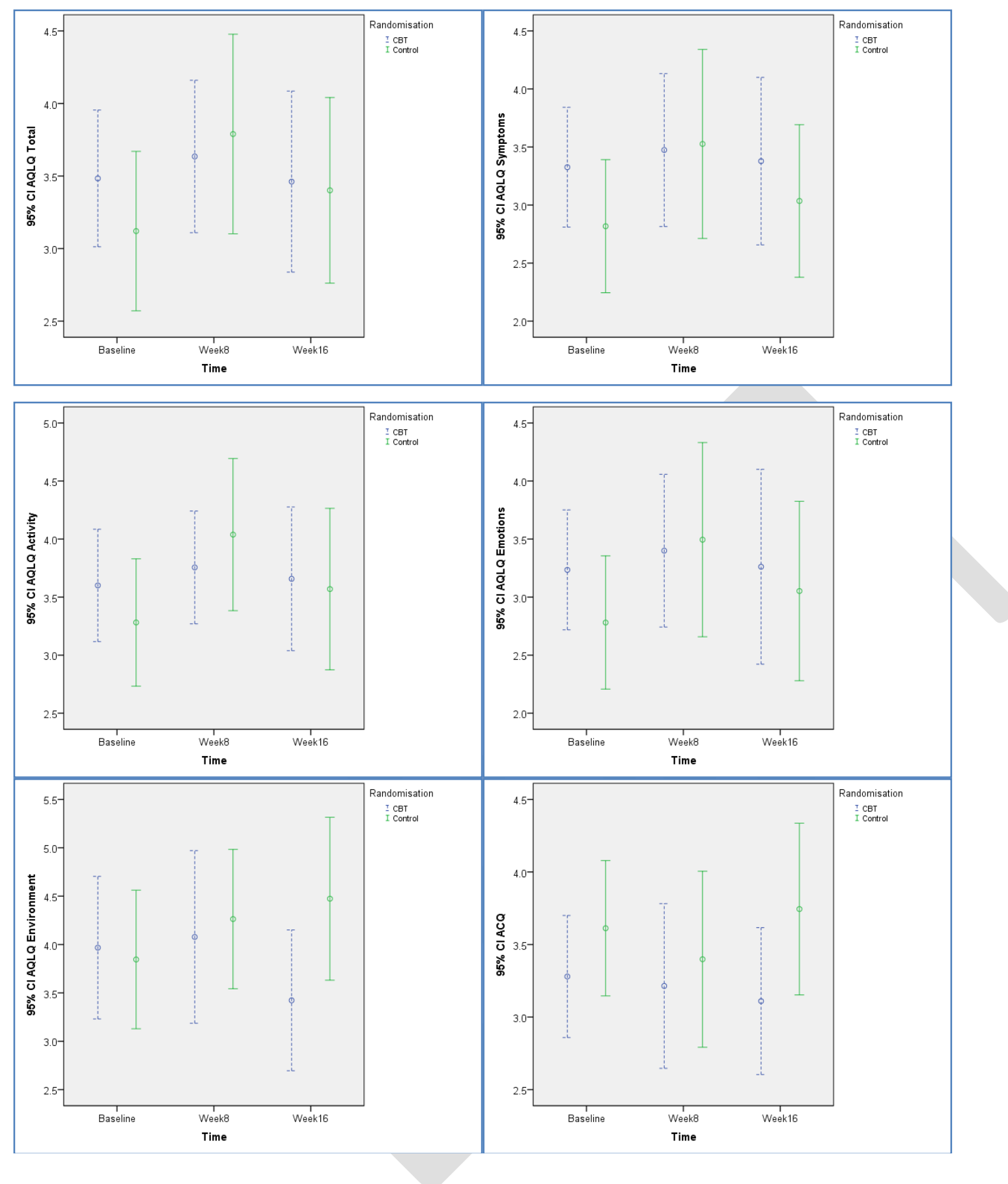

19 

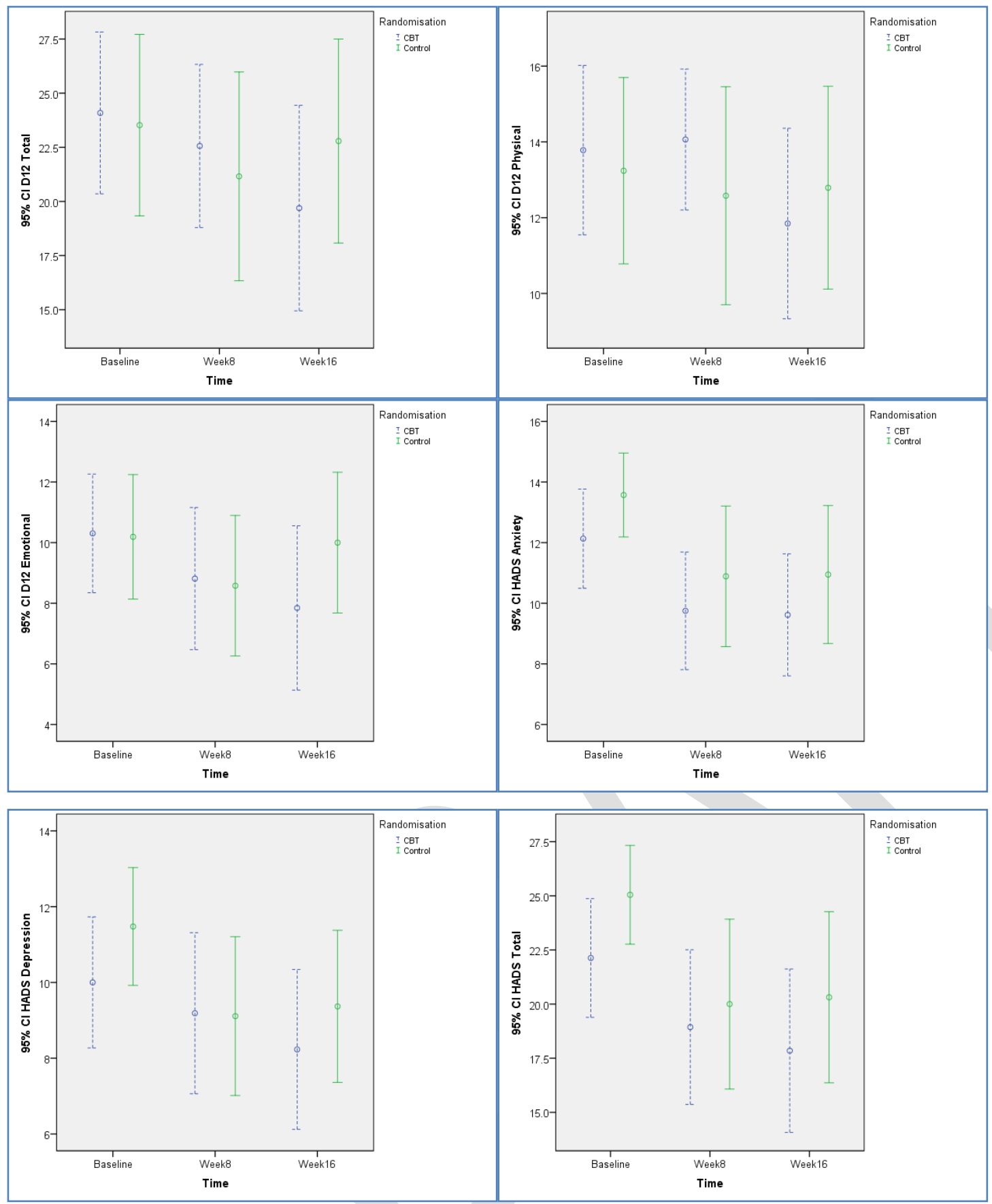

Figure 2: Mean scores $(95 \% \mathrm{Cl})$ from baseline to 8 and 16 weeks. AQLQ symptoms, AQLQ activity, AQLQ emotion, AQLQ environment, AQLQ Total, ACQ, D-12 Total, D-12 Physical, D-12 Emotional, HAD Anxiety, HAD Depression, HAD Total 
Figure 1: Consort Diagram

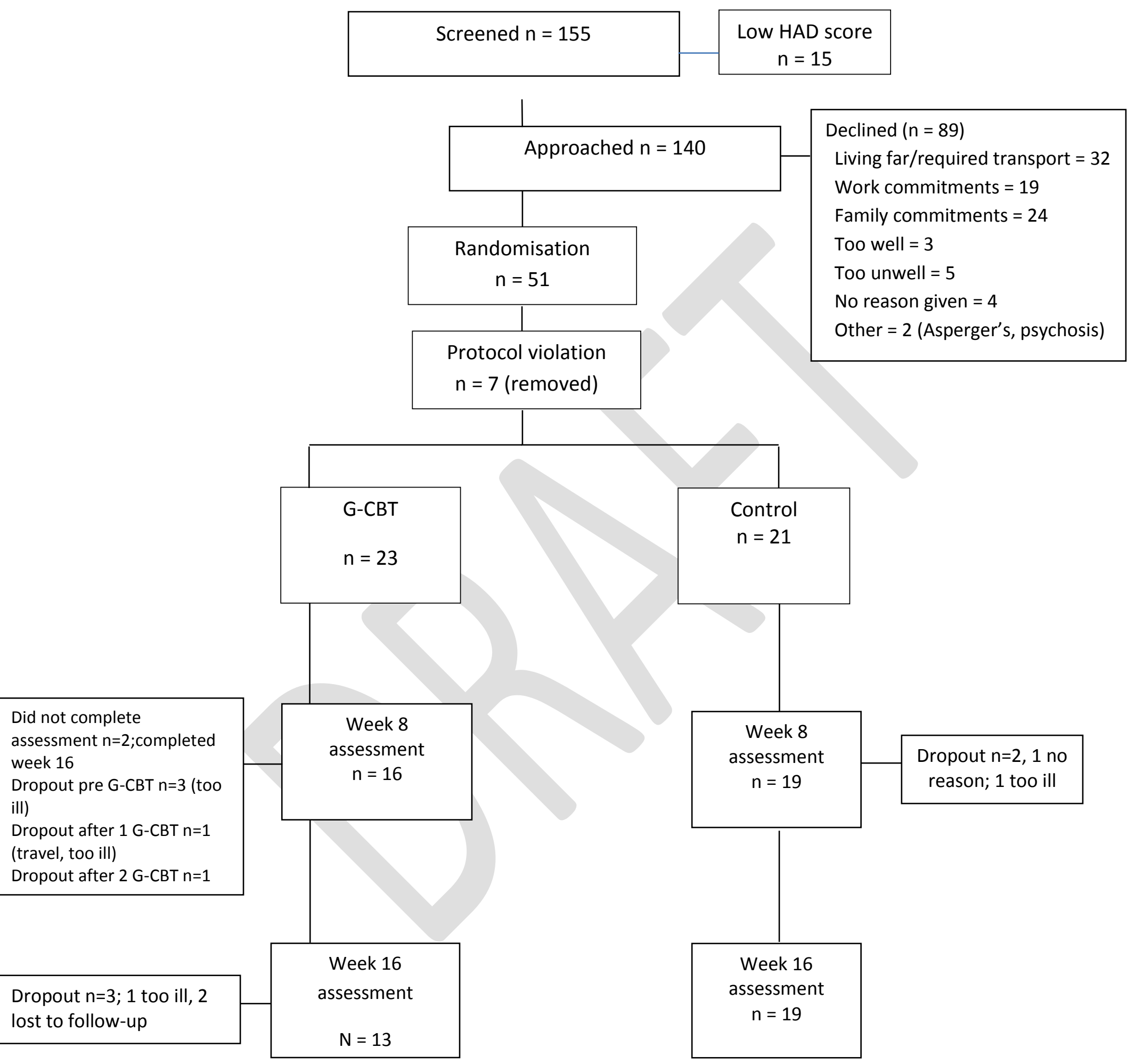

